\title{
State of the Art Report - A Comparative Study of Structural Analysis and Design Software - STAAD Pro, SAP-2000 \& ETABS Software
}

\author{
Balwinder Lallotra ${ }^{\# 1}$, Dhirendra Singhal $*^{2}$

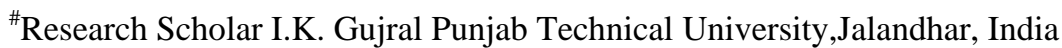 \\ 1balwinder.com@gmail.com \\ *Professor and Chairperson, Department of Civil Engineering, \\ D.C.R. University of Science and Technology, Murthal, India \\ 2singhald62@rediffmail.com
}

\begin{abstract}
The aim of this paper is to conduct an in depth study of the major features of three structural analysis and design software programs STAAD Pro, SAP-2000 and ETABS software which are generally used. Each software program has its own features, analysis option, design and output options, limitations and advantages. It is necessary for the user to exploit all the advantageous feature of particular software program but also the software program should not be used beyond its applicability to avoid any catastrophic failure. Therefore, this paper discusses the main features of all these software programs with the emphasis on applicability and limitations of these software. This will enable a designer to choose required software according to the requirements of a structure with confidence.
\end{abstract}

Keywords- Advantages, Limitations, ETABS, SAP-2000, STAAD Pro.

\section{INTRODUCTION}

The construction in the present era is becoming challenging day by day in order to achieve more economy and efficiency. The designers as such require better skills and sound knowledge. Such challenges can be tackled more efficiently by using computer based software programs available in the market developed by different software organizations. However, these software programs cannot offer universal solution to every problem.

The structural analysis program is divided into two categories: general purpose software program [1] and design oriented software programs [2-4,6]. The general purpose software programs are used for a wide range of scientific and engineering purpose. The general purpose software programs commonly used are Abaqus and ANSYS. Abaqus [9] are more advanced with advanced solver (implicit or explicit solver) suitable for computing all types of complex problems in engineering and sciences. The implicit solver integration method is ideal for the solution of static problems and the explicit integration is suited for solution to dynamic problems such as fire, blast and temperature variation. The general purpose software programs are suitable to analyze wide variety of problems such as uncertainties in loading and materials using finite elements. ANSYS [1] is used for all most all engineering disciplines, with application to static load, dynamic load on wide range of material models. It supports both linear as well as nonlinear analysis with nonlinearity in material (i.e. plasticity) and geometric non linearity (e.g. cable structure). The general purpose software programs are more sophisticated and accurate in terms of mechanical modeling and geometrical capabilities. However these software has a limited use in civil engineering problems, as specific civil engineering functions are not available which requires further improvements in graphical user interface (GUI). One of the biggest disadvantages of the general purpose software programs is that the design checks as per code cannot be performed in such packages which is urgently required for solving civil engineering problems. To handle large structures computers with very high configuration is required and time taken for the analysis is very large (in hours and days for analysis depending on the size of project handled). The results produced by the general purpose design software program require additional processing before they are useable in structure design. All these drawbacks make the general purpose software unsuitable for commercial use in civil engineering. The use of general purpose software is restricted to researcher work and elements design only.

The design oriented software programs [2-4] are tailor made software, developed specifically for a solution to specific problems. These software programs such as STAAD Pro, SAP-2000 and EATBS are pre-programmed to give quick results according to the code of practice. For example if a multistoried building is modeled using different structural elements such as beams, columns, slabs with different loading and supports restraints. After analysis an engineers can get bending moments, shear force, axial force and deflection of the structural members of the whole building. The design check to find the correct size of the structural member is also available in the design oriented software. The design oriented software provides the solution to large structures 
with desired specific results such as area of steel, concrete quantity and size of the element required to make the structure safe. The design oriented software uses limited number of finite elements as a result, the analysis time is bare minimum as compared to general purpose software. These software can easily handle large multistoried projects with ease.

The design oriented software are therefore more widely and accepted software used nowadays for the solution to civil engineering problems by the designers and infrastructure developers.

\section{NOTABLE DIFFERENCES IN FEATURES OF STAAD PRO, ETABS AND SAP-2000 SOFTWARE}

This paper aims to discuss the features of three state of art modeling software programs STAAD Pro, SAP2000 and ETABS which are commonly used [2-7]. This attempt will provide much convenience to designer to enable him to gain confidence to choose software program according to the requirements of a particular problem which may enable the designer [8] to provide more accurate and economical solutions.

\section{A. User Interface}

STAAD Pro, SAP-2000 and ETABS follow the right hand rule for the Cartesian coordinate system in X, Y, Z direction. These entire axes are at right angle to each other and are used to define the location of joint and direction of loading. In STAAD Pro X-axis, Z-axes are taken in plan and Y-axis is taken vertically upward shown in Figure 1. In SAP-2000 and ETABS software X-axis, Y-axes are taken in plan and Z-axis is in vertical direction. These are shown in Figure 2 and Figure 3 respectively. These axis are used to locate the position of the nodes and elements on the structure when the structure is modeled. According to the experience of the author the directions of the Cartesian coordinates followed by STAAD Pro software is more convenient to the user as height in engineering is taken along Y-axis and these directions are globally adopted for specifying the coordinates.

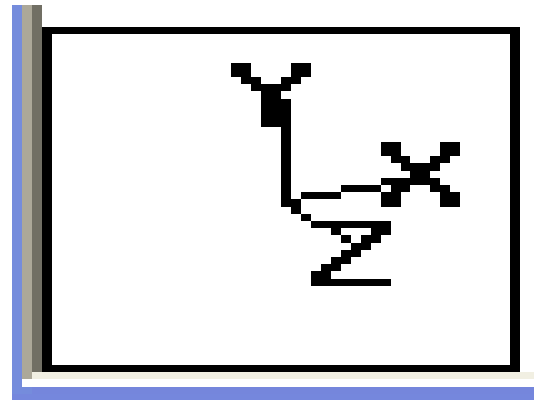

Fig.1. X, Y, Z-axis in STAAD Pro

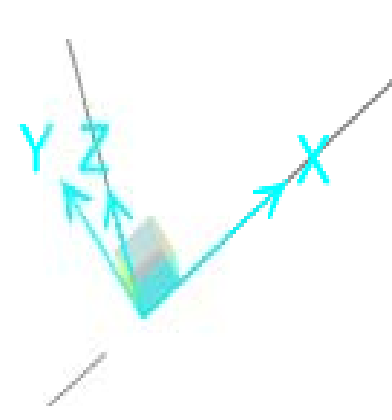

Fig.2. $\mathrm{X}, \mathrm{Y}, \mathrm{Z}$-axis in SAP-2000

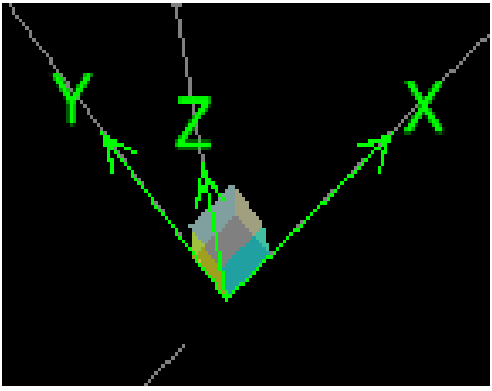

Fig.3. $\mathrm{X}, \mathrm{Y}, \mathrm{Z}$-axis in ETABS

STAAD Pro [2,6] software program has a bold single window graphical user interface (GUI) to indicate the control as shown in Figure 4. The tools available in the graphical user interface are used to perform different functions to run the software. The STAAD Pro has STAAD editor page which is of great help in modeling structure. Any change in the model can be done using the editor page. This feature in STAAD Pro makes it the most versatile software to be used by the engineers. The model in STAAD Pro can be edited using either Graphical user interface or by editing in STAAD editor file. The STAAD editor page has a color coded tool to label, organize and check comments about the model which helps to organize the stage construction. These features are not available in SAP-2000 and EATBS software program.

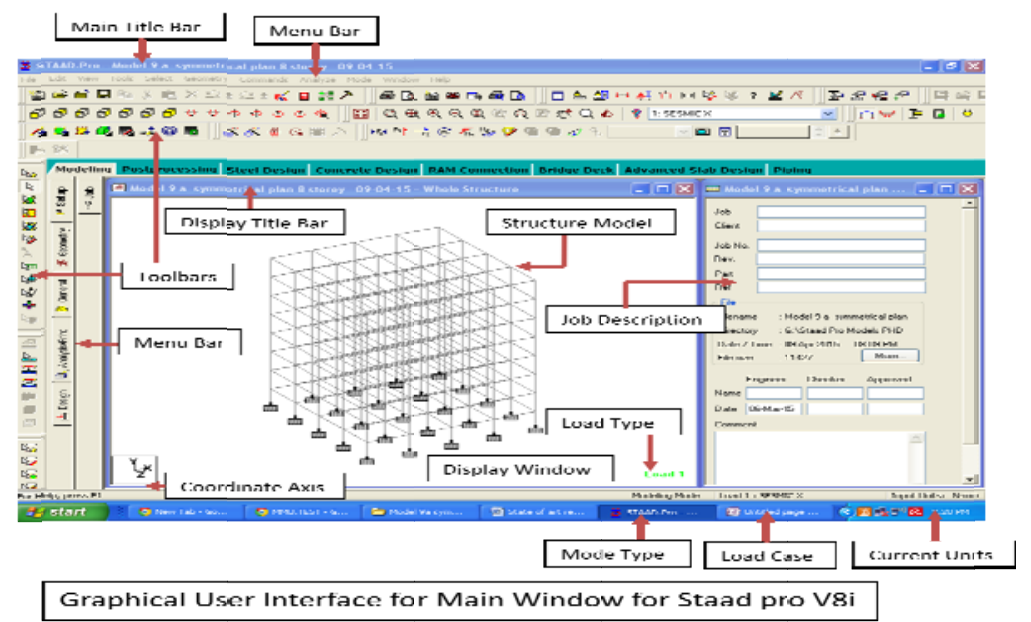

Fig.4. Graphical User Interface in STAAD Pro software program 
SAP-2000 [3] is design oriented software program for design of bridges and buildings. It has a single user interface to model, analyze and design to get the output results using customizable window shown in Figure 5. SAP-2000 has a powerful user interface to design, display and analyze any type of structure with the help of grid which can be edited as required to suit the plan of a building. User has a flexibility to see the different views using dockable windows. One to four windows can be displayed at a time with different views. The tasks such as material properties, sections, mass source, coordinate system/grids, joint constraints, joint patterns, functions (Response spectrum, Time history), load patterns, load cases, load combinations, moving loads, Pushover parameter sets are grouped under a common heading "Define” menu.

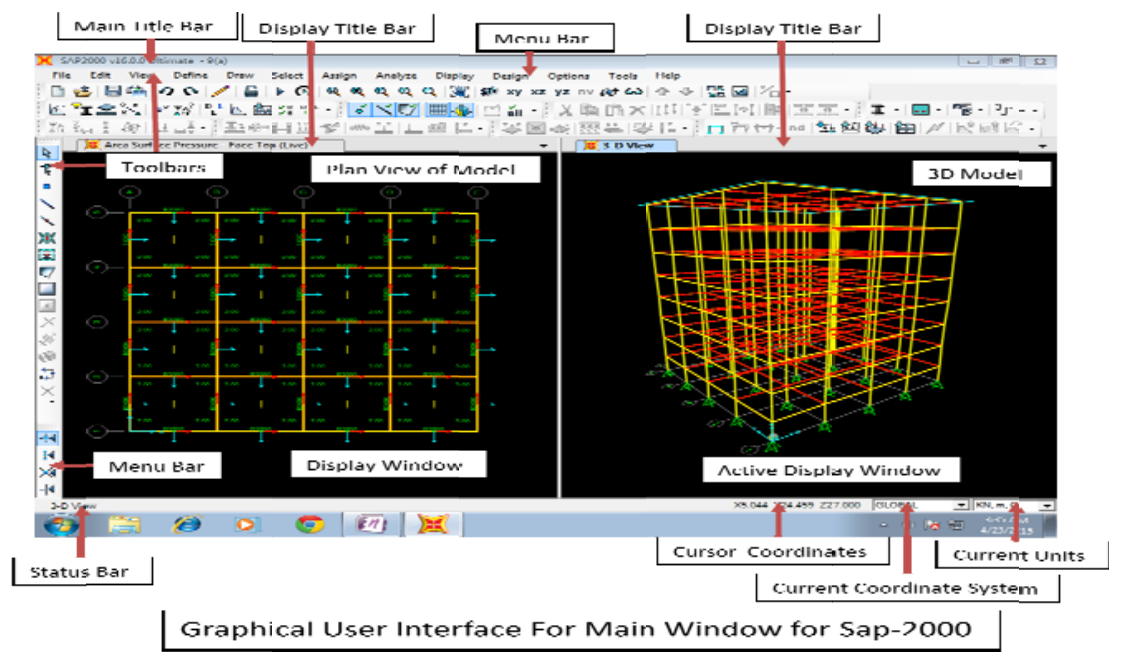

Fig.5. Graphical User Interface in SAP-2000 software program

ETABS [4] stands for the extended three dimensional analysis of building structure. This is a design oriented software program specially built for the analysis of multistory buildings fully integrated with simple, intuitive window based user interface shown in Figure 6. ETABS has a smart start and all tasks are grouped under "define" menu such as material properties, frame sections, wall/deck/slab section, diaphragms, response spectrum functions, time history functions, and static linear/pushover cases, sequential construction case, load combinations, design combos. The graphical user interface is great as it can generate 3D model using plan and elevation. Views up to four windows are possible simultaneously. The graphical user interface (GUI) in ETABS is more user friendly for modeling a multistoried buildings compared with graphical user interface (GUI) of STAAD Pro and SAP-2000 software program.

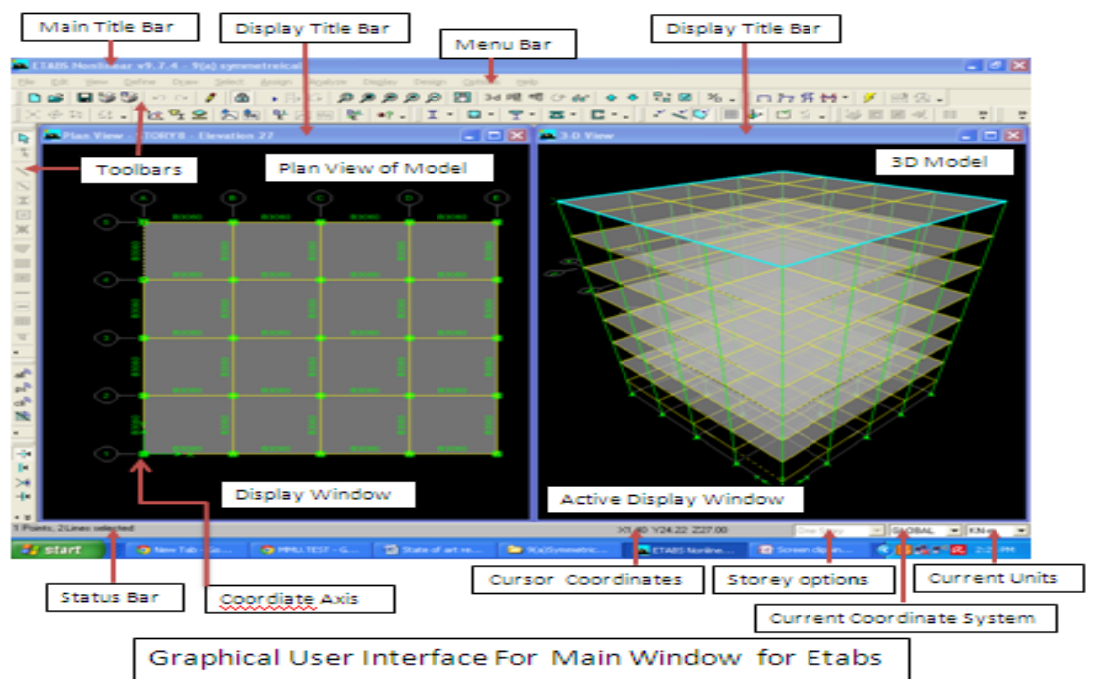

Fig.6. Graphical User Interface in EATBS software program 


\section{B. Modeling Features}

Modeling is an important aspect which must be taken care to represent the actual behavior of the structure. The accuracy of the software results depends on how accurately the modeling is done to represent the actual behavior of the structure keeping in mind the type of problem to be analyzed. Therefore, it is very important for the user to pay special attention to modeling aspect of the structure.

The key to choose a suitable analysis program in design practice is based on specific engineering problem. This is particularly important as the complexity of the analysis increases; certain package may be more suited to a particular application. Therefore different modeling features of all the three software are discussed.

STAAD Pro $[2,5,6,8]$ has a main modeling features grouped in geometry menu and commands menu. The tasks such as creating beam, plate, split beam, insert nodes, mirror, renumber beams, renumber nodes, translational repeat, circular repeat, generate surface meshing, move and rotate are very helpful in modeling the structure. The structure shown in Figure 7(a) is modeled using these features. The other unique feature in STAAD Pro such as translational repeat, copy and mirror are very helpful to model a complex structure with fewer efforts. Mirror and translational repeat command are used to model a structure of a steel space vault as shown in Figure 7(b) and Figure 7(c). Nodes can be created on the beam for modeling secondary beams at the specified location by just adding the distance in edit node option. These nodes can be moved to a required position by specifying the distance in move command available in "geometry" menu. The tasks grouped under "commands" menu are material property, boundary conditions and load combination for the modeled structure. These properties are assigned to a modeled structure so that real structure behavior can be represented. The model is created using structural grids or by simply adding coordinates of single floor and then adding beams to these nodes. The model can also be created using STAAD editor page easily. Modeling and input data is stored in a text file with extension (file extension.STD). Any change in the model can be made by editing the data in this extension file or by using the graphical user interface. It has an interactive menu driven model generation with simultaneous 3D display. The predefined modeling templates available in structure wizard command are truss models, frame models, surface plates, solid box, silos and bunkers which are very helpful to model complex structures. The software program is compatible to import models from CAD drawings to STAAD Pro software program. Recently the STAAD Pro is integrated with special feature of application programming interface (API). OpenSTAAD is a library of exposed functions allowing the user to access internal routines and function of the software program through API for personal customization. The output and the input in STAAD Pro can be directly linked to third party application using any programming language such as $\mathrm{C}, \mathrm{C}^{++}$, FORTON, VB, VBA and Delphi through OpenSTAAD library. The user can write their own VBA based customized macros using inbuilt VBA editor, to have access in controlling the view, geometry, member specifications, loads and result display. The macros are very helpful to automate the repetitive tasks. For example a simple macro can be written in excel or within the STAAD Pro software program using openSTAAD which automatically links with the software output results. Any change in the STAAD file changes the results in the output file with the built-in macros. The input data in STAAD Pro has to be entered in a sequential and logical way otherwise it will not perform the analysis or it will give errors. For example if the member loads are added before the floor load the STAAD Pro will not perform the analysis therefore it is necessary to add the floor load before the element load is assigned to the structure which can create confusion to a new user.

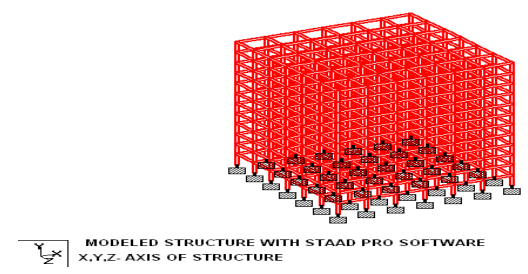

Fig.7.(a) 3D Structure model in STAAD Pro

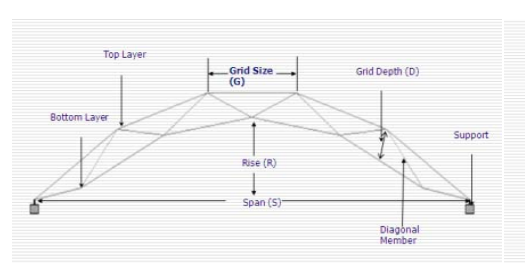

Fig.7(b) Front View of space vault

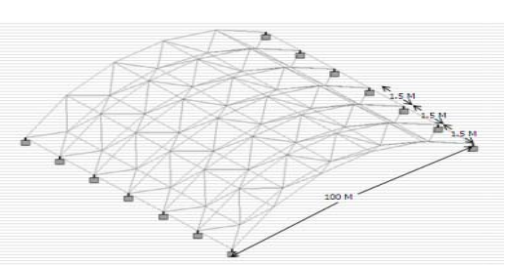

Fig.7(c) 3D view of space vault

SAP-2000 [3, 5] has intuitive graphical user interface (GUI) with realistic modeling shown in Fig 8(a). This software program has a wide selection of parametric templates for different types of structures such as 3D frames, trusses, staircases, Dam structures, shells and pipes which makes the modeling easy fast and is more suited for the analysis and design of such structures. Some of the structures modeled using the predefined templates available in the software program are as shown in the figure 8(b-g). 

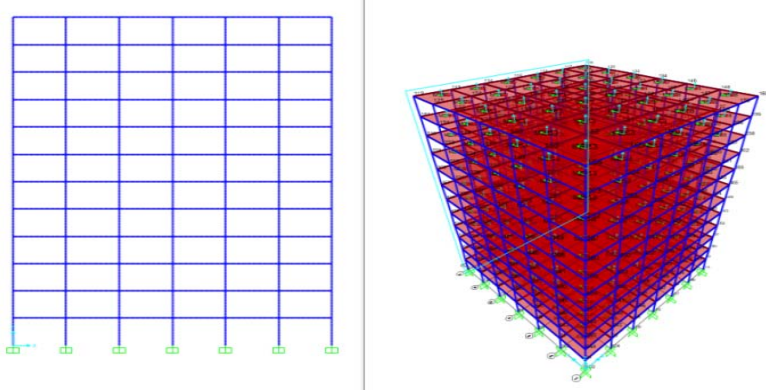

Fig. 8(a) Structure modeled in SAP-2000 with two windows (Elevation \& 3D)

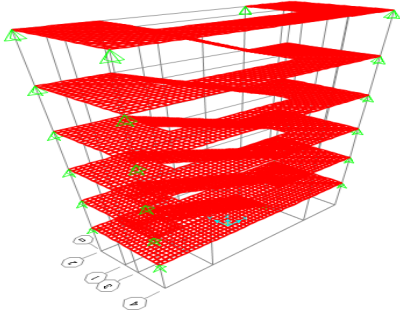

Fig.8 (b) Stair case

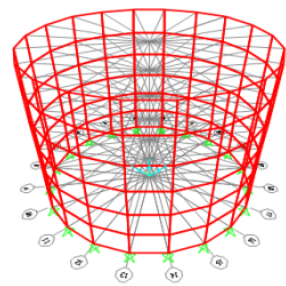

Fig.8 (e) Shell structure

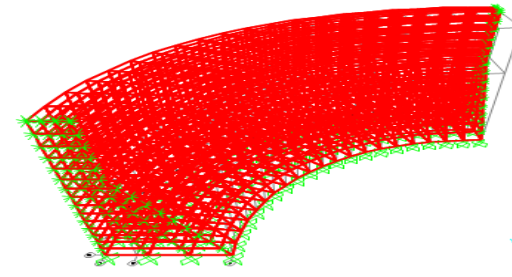

Fig.8(c) Arched Dam (plan)

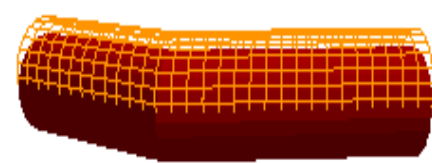

Fig.8 (f) Model of a pipe bend

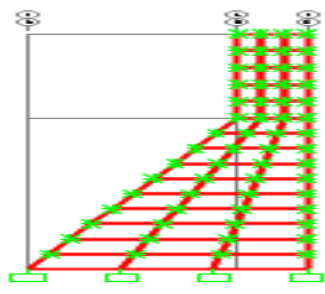

Fig.8 (d) Arched Dam (x-section)

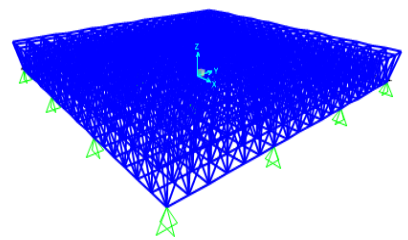

Fig.8 (g) Space truss

In SAP-2000 [3,5] grids are defined as a Cartesian or cylindrical with no limit to grids. These grids can be rotated in any direction to model a structure such as circular water tank, dome etc with ease. Plan and elevations are automatically generated at every grid line to get model quickly. SAP-2000 engine automatically converts the assembly into finite element by meshing the area into smaller elements and sub-elements. Mismatched elements are matched by joint interpolation algorithms edge constraint technology which acts as zipper between the elements. The shape is further refined by mesh reshaping tool available in software program. Almost any type of structure can be modeled and analyzed using SAP-2000 software.

ETABS [4,5] is a design oriented software program specially built for modeling and analysis of multistoried structure as shown in Figure 9(a). One of the advanced and powerful features available in ETABS software is "similar stories" feature to duplicate the work at typical stories. Once this feature is activated, any change made in the plan at master story is replicated at all the typical stories. Modeling of complex structures can be done fast as it has an option of working in single storey, all stories and by selecting stories with similar plan to the master storey. All input data can be assigned simultaneously to all similar stories reducing the man hours of work making project more economical. This feature is not available in STAAD Pro and SAP-2000 software program. Each column has one number for all stories and similarly placed beams at different levels with same beam numbers shown in Fig 9(b). This reduces the large number of elements in a complex multistoried structure to few element groups for design.

The ETABS is purely building design software and more suited for analysis of large structures. The modeling in ETABS is easier as elements (columns and beams) in this program are assigned in plan i.e. 2D whereas the modeling in SAP-2000 is very tiresome as elements (columns and beams) are assigned in 3D. SAP-2000 is more suited for analysis of small structures or a portion of the large structure with regard to its meshing options. Design program in ETABS and SAP-2000 automatically calculate the clear size of the elements (beams and column) based on the size of the members and gives realistic feeling to the user for better confidence but this facility is not available in STAAD Pro software. STAAD Pro is equipped with programmable VBA macro for personal customization but this feature is not available in ETABS and SAP-2000 software program. The result interpretation in ETABS and SAP-2000 is very easy as compared to STAAD Pro as the design results are directly displayed on the elements graphically. The columns and beams are easy to identify in ETABS as compared to SAP-2000 software since ETABS software program has a common labeling at all the floors but 
this process is tedious in case of STAAD Pro software program as each element (columns \& Beams) are given unique identity as shown in figure 9(c) and 9(d). Therefore the sorting beams and columns in certain groups are required to make the results more practical for making the drawing for execution of the project. The properties in STAAD Pro are assigned after the structure is modeled which requires the careful assignment in complex structures as missing out any member can give the disastrous results. The chances of such error are less in case of SAP-2000 and ETABS software programs because the properties in these software are assigned to each element before these are added in the frame to make a structure. SAP-2000 has the capacity to model and design complex structures such as retaining wall, overhead water tank, underground water tank, pile cap, steel space structure, even the bridge can be modeled and designed with this software program. Isolated footing can be modeled and designed in STAAD Pro and SAP-2000 software program but not possible in ETABS software program. The structures shown in the Fig (8b, 8c, 8d and 8g) can also be modeled using STAAD Pro program but its modeling requires more efforts for modeling in STAAD Pro than SAP-2000 program as these are available in the predefined templates in the program itself. These structures cannot be modeled and analyzed in EATBS software program. In STAAD Pro all the data is entered in a sequential and logical way but this is not necessary in ETABS and SAP-2000 software programs making it more convenient for the new user.
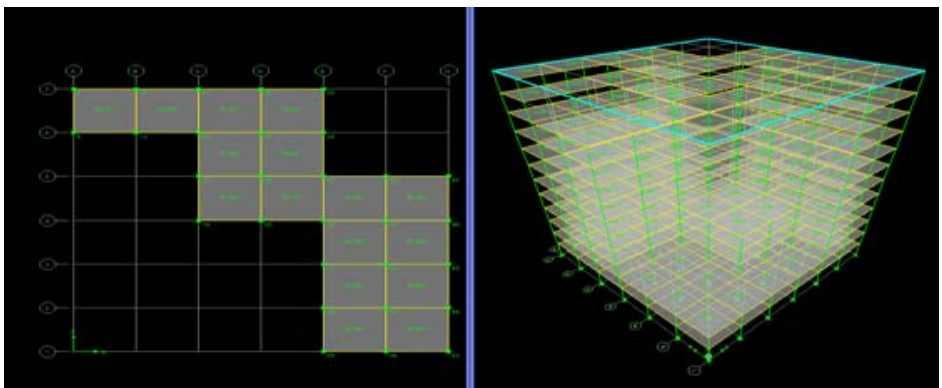

Fig.9 (a) Structure modeled in ETABS with two windows (elevation\& 3D)

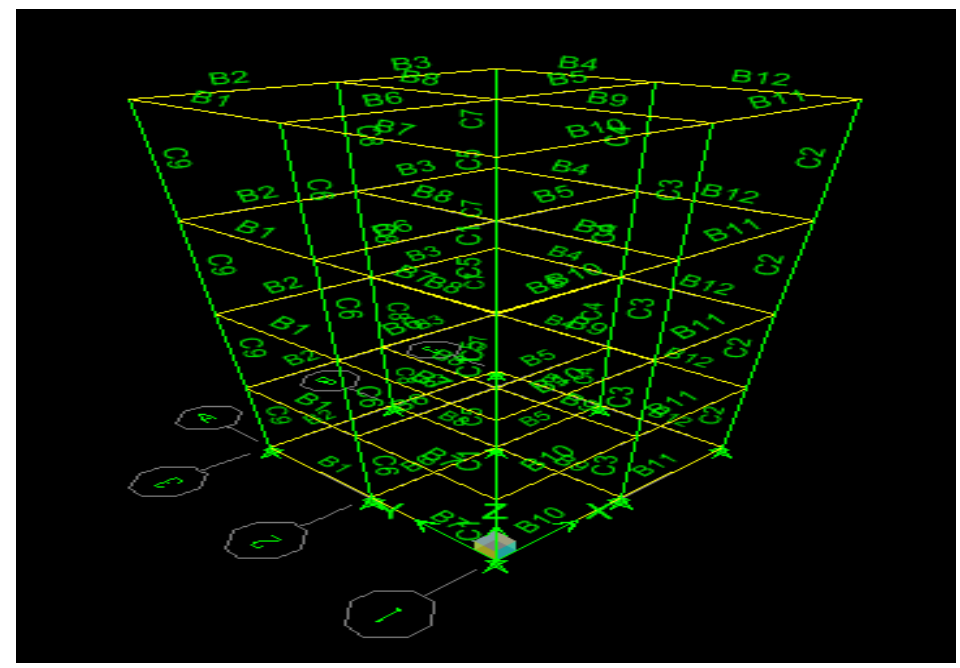

Fig.9 (b) Structure in ETABS with common labeling at different floors

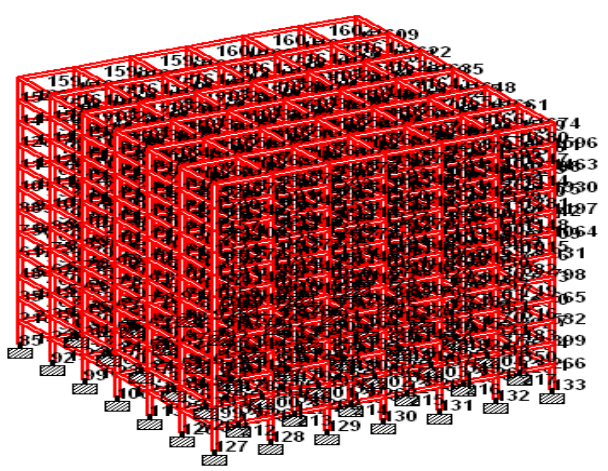

Fig.9(c) Structure in STAAD Pro with elements labeling at different floors 


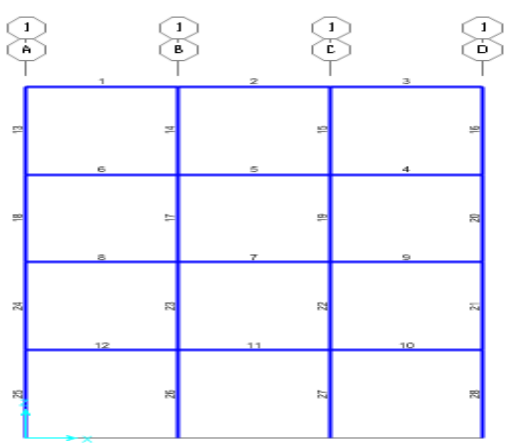

Fig.9 (d) Structure in SAP-2000 with elements labeling at different floors

\section{Vertical Loads}

The basic principle of transfer of load in the structure is same in all the three software. The vertical loads applied on the structure are self weight, superimposed dead loads, live loads, reducible live and snow loads. Self weight of the structure is generated by the programs itself from the weight density assigned in the software program and acts in the direction of gravity. The programs use the self weight as a uniformly distributed member load in analysis.

The vertical load is taken along Y-axis of the structure in STAAD Pro software program. Load acting vertically upward is taken as positive and vertically downward direction is taken as negative. Vertical load in STAAD Pro is applied to node, line elements and object areas shown in Fig. 10. Line elements can be assigned linear loads, concentrated loads, uniformly distributed loads, trapezoidal loads and triangular loads in the direction of gravity. Floor may be given uniform load or area load which may depend on one way or two ways slabs. STAAD Pro software program cannot add the self weight of the slab in a structure as it has to be calculated and added to the vertical dead load. The floor load in STAAD Pro can be directly provided without assigning the floor slab to the framed structure by providing the maximum and minimum limit to the floor slab. The area load is converted into load per unit area on member load based on one way or two way actions. STAAD Pro gives warning "Load losses” if the plate releases are poorly defined.

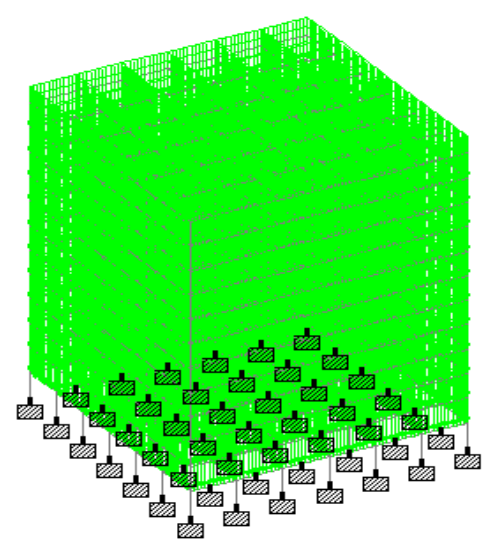

$\sum_{Z} \mid \begin{aligned} & \text { DIRECTION OF GRAVITY } L \\ & X, Y, Z \text { AXIS OF STRUCTURE }\end{aligned}$

Fig.10 STAAD Pro with Gravity Loading

In SAP-2000 [3] the frame can be assigned vertical load which may be applied to the joints, frame element and on slab as area load as shown in Figure 11. These are provided in the direction of gravity i.e. along Z-axis of the structure. Load assigned in upward direction along Z-axis is taken as positive and load assigned in the direction of gravity i.e. along Z-axis direction is taken as negative. The vertical floor loads in SAP-2000 can only be provided by modeling a floor as a plate element and the meshing it to create a bending action. The floor transfers the loads applied normal to the plane by bending action of the floor. 

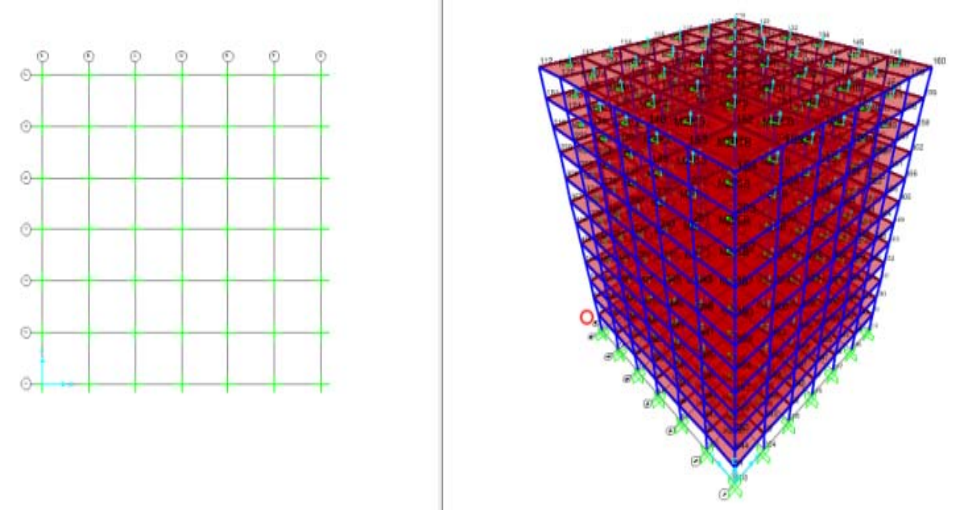

Fig.11 Direction of gravity load in Z-direction (vertical) in SAP-2000

In ETABS [4, 7] the loads may be applied to point, line and area objects in the direction of gravity shown in Figure 12. Concentrated forces or moments can be applied to point load. Vertical load applied in the upward direction along Z-axis is taken as positive and in vertically downward direction is taken as negative. Framed objects may have number of point loads or distributed loads. Uniform loads can be applied to area object. ETABS transfers the load on the members supporting the floor plate by bending action. Floor loads can be applied by selecting the objects graphically even for irregular panels there by automating the tedious work of transferring the tributary load of floor to the adjoining beams.
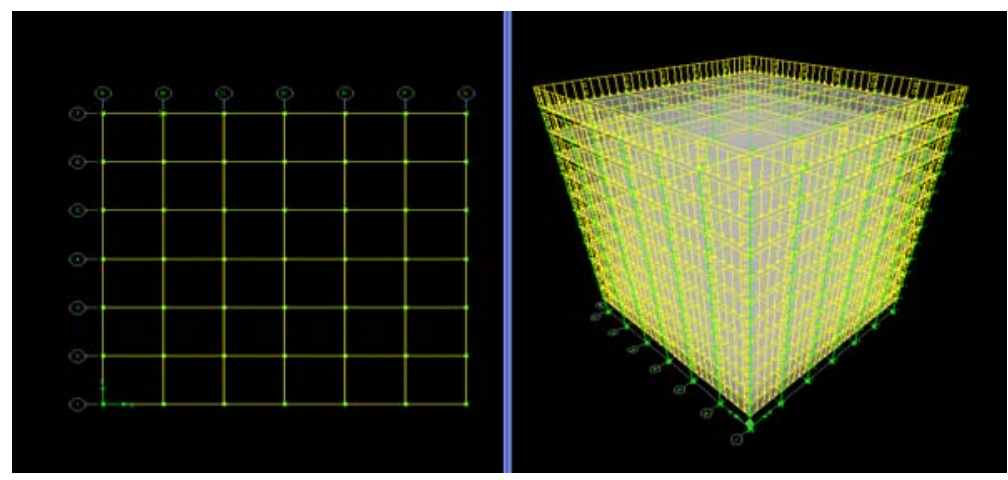

Fig.12 Direction of gravity load in Z-direction (vertical) in ETABS

STAAD Pro has the advantages that the slab load can be directly assigned to the structure by providing the minimum and the maximum value of Y-coordinate in a floor load assignment. There is no need to model a plate to transfer the load to the elements of the structure which saves modeling time. The floor load in ETABS and SAP-2000 can only be provided if the slab is modeled as a plate element and meshed to transfer the load to the framed element which is time consuming and tedious work.

\section{Lateral Loads}

Wind and seismic loads in STAAD Pro [2,6] is provided in X-direction and Z-direction (Y-axis being vertical in STAAD Pro) shown in Figure 13(a) and Figure 13(b). The base shear is automatically calculated by the STAAD Pro software program using appropriate equations from building design code of the particular region. The base shear or the total lateral seismic forces are distributed at various floor levels according to design code specified by the user and then these loads are utilized for analysis and design. The software program has the capability to generate the wind load as per the various international and domestic building codes integrated with the software. The openings in the structure are modeled by giving exposure in fraction. The frame consisting of column, beam and slab resist the lateral load acting on the structure by rigidity of the structure. 


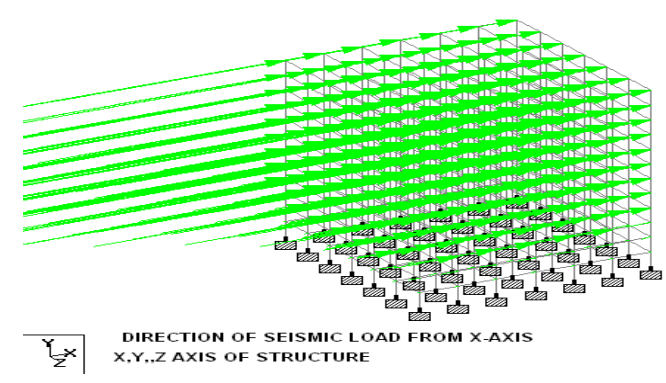

Figure 13(a) Structure with lateral load in X-direction in STAAD Pro

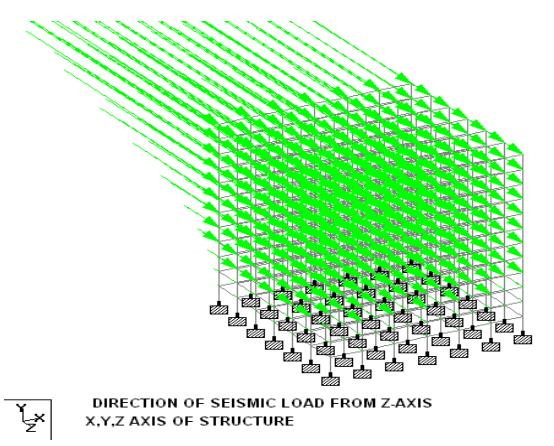

In SAP-2000 [3] software seismic and wind load is automatically generated and assigned to a structure as per the different international and domestic codes integrated in the software shown in the Figure 14. Lateral loads in SAP-2000 are based on the specified mass of the structure. Once the lateral load is calculated, the load is transferred to each point at different levels of the storey in proportion to its mass. SAP-2000 takes the eccentricity in case of rigid diaphragm and also includes it for calculations of lateral loads in the moments in rigid diaphragm. Earthquake and wind load can be assigned to SAP-2000 which takes care of reversal of stresses (positive or negative stresses). This software has a sophisticated moving load generator for frames and shells. Software has wave-loading feature to generate load on the structure due to wind, buoyancy, current flow resulting from multi-stepped static and dynamic loading to include inertial effects of moving load through the structure. SAP-2000 [3] has a special feature of joint pattern which transfers the lateral load on the structure. Joint pattern is a set of scalar quantity defined at the joint. Joint pattern is referenced when assigning pressure and temperature loads on the structure. This is the most versatile feature of SAP-2000 for the distribution of complex pattern of load distribution on the structure. Joint pattern itself does not provide any load on the structure. The joint pattern definition and assignment defines the variation of temperature for area objects and variation of temperature for the frames. The software also has inbuilt design code which automatically generate seismic and wind loads to the structure.

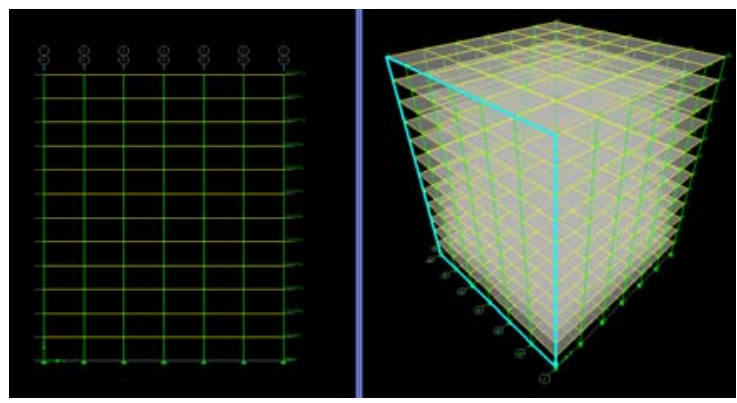

Fig.14 Structure with lateral load in X-direction in SAP-2000

Fig 15 shows the lateral loads such seismic loads in ETABS is automatically calculated from the member dimensions and built in sections integrated in the software supporting various codes of different countries. The dummy area is provided on the sides of the building to calculate the wind load in ETABS. Using this dummy area and the wind pressure coefficients based on selected code, the wind load is automatically calculated by the ETABS software program. The wind loads in rigid diaphragm is applied the geometric centers of each rigid floor diaphragm. In case of semi-rigid diaphragm ETABS uses special load dependent Ritz-vector algorithm for quick automatic calculation of predominant time period.

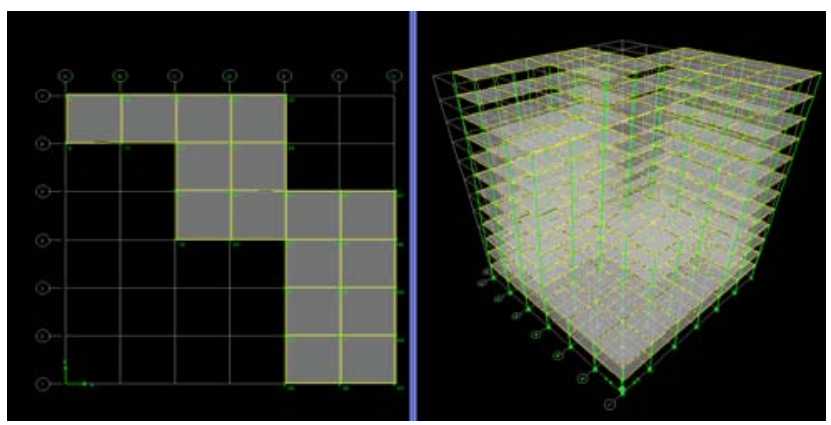

Fig.15 Deformation due to seismic load in Z-direction in ETABS 


\section{E. Analysis Option}

STAAD Pro [2,6] is a design oriented state of art software program in which stiffness analysis is based on matrix displacement method. It is capable to analyze any type of structure such as multistoried framed buildings, towers, bridges, industrial buildings and utility structures ranging from $1^{\text {st }}$ order static to $2^{\text {nd }}$ order Pdelta analysis both large and small P-delta including stiffening effects. STAAD Pro is capable to do dynamic analysis, time history analysis, modal analysis and response spectrum analysis both for linear and nonlinear analysis. Software supports various codes such as concrete, steel and timber of various countries. STAAD Pro has basic solvers and advanced solvers. The advanced solver is 10 to 100 times faster, uses less memory and the files are highly optimized.

SAP-2000 [3,7] has a very innovative technique SAPFire engine to give optimum analysis with multiple 64 bit solvers. SAP-2000 has advanced sophisticated analysis for dynamic and non linear consideration. This feature makes SAP-2000 the most versatile and practical tool suitable for analysis ranging from static linear and nonlinear construction sequence loading, dynamic response spectrum which gives the pseudo spectral acceleration with periodic curve, dynamic linear and nonlinear time history analysis, large displacement analysis, Bridge analysis and buckling analysis. SAP-2000 option includes Eigen analysis and Ritz analysis (auto shifting of ill conditioned relations and convergence criteria respectively). Buckling analysis provides the information of linear buckling i.e. about different mode shapes under non linear static and dynamic application. Non linear analysis considers P-delta and large deflection effect for the analysis.

ETABS is developed mainly for the buildings frames. The software is suitable for doing sequential construction, response spectrum analysis, time history analysis, P-delta analysis, and pushover analysis. ETABS has advanced computational algorithms for analyzing a complex high rise structures easily in a shorter time. It has option to take the effect of moments and forces into account due to P-Delta effect. The user has an option to turn it off or on as per their requirement for the type of structure. ETABS has special feature of bar arrangement as the input to get the realistic and economical design. Design of any arbitrary shape column is possible using this software. Intuitive and customized member properties are of great help during checking and revision of model through different inbuilt codes. The numerical analysis technique used in ETABS has been discussed in Professor Wilson's book titled, “Three dimensional static and dynamic analyses of structures". The ETABS software program has a powerful numerical procedure with advanced algorithms for fast calculations using less memory.

\section{F Design Options}

STAAD Pro is capable of doing concrete design, steel design, timber design and aluminum design. The software gives the optimum design section on giving "select Optimized" command for steel structures using an optimization technique based on multiple analyses. To achieve this program performs number of iterations as well as analysis to get economical design. The output is given in the output file which is printable in a format given in figure 16(a-c) and graphically shown in Fig 16(d). The STAAD Pro input file is processed by STAAD engine and the data is stored in different files. The results of extension files are used by graphical user interface (GUI) in the post processing mode.

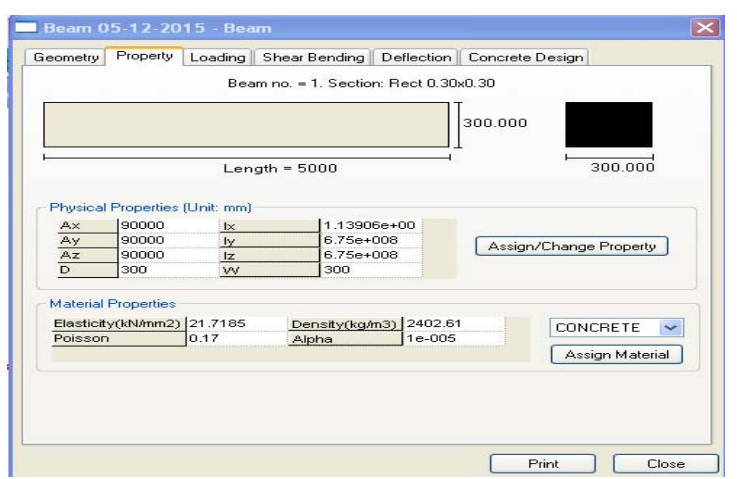

Fig.16 (a) Detail showing member properties

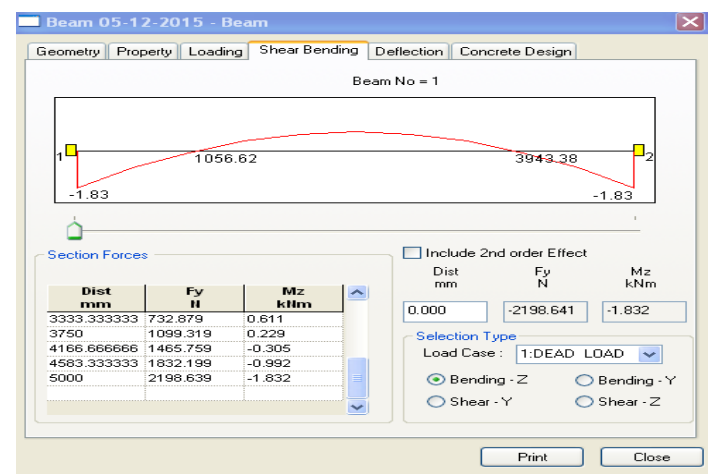

Fig.16 (b) STAAD Pro Bending moment output 


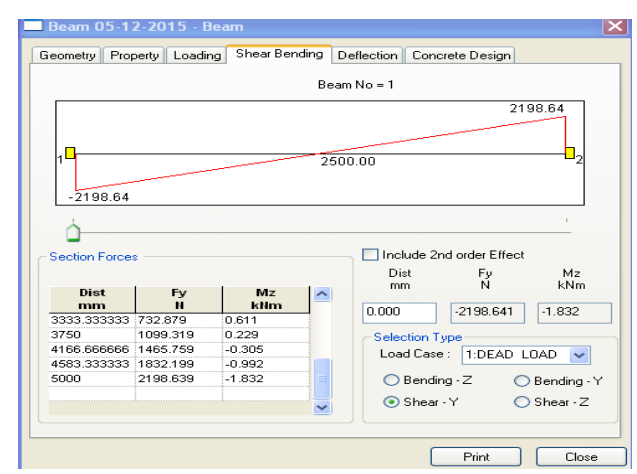

Fig.16(c) STAAD Pro Shear force output

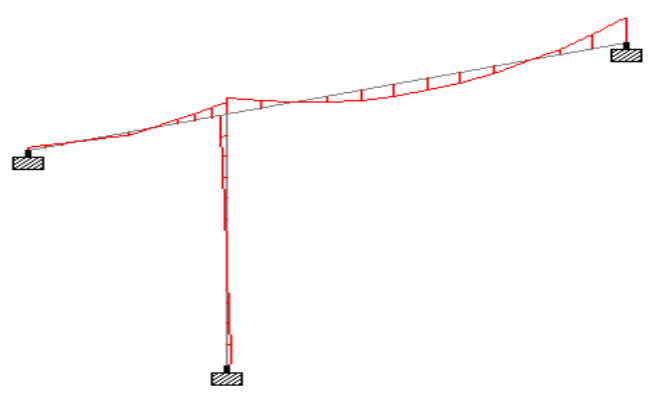

Fig.16 (d) Graphical representation of B.M. \& S.F. in STAAD Pro

SAP-2000 [3, 7] is fully equipped with feature to do steel design, concrete design and aluminum frame design supported with inbuilt design codes as per international standards. The SAP-2000 has option to do static and dynamic loads analysis with ductile and non ductile detailing of steel reinforcement. Column can be designed for axial load and biaxial moment. The output detail of the SAP-2000 gives the results in the form of shear, moment and deflection as shown in the Figure 17(a) and its graphical representation is shown in the Figure 17(b).

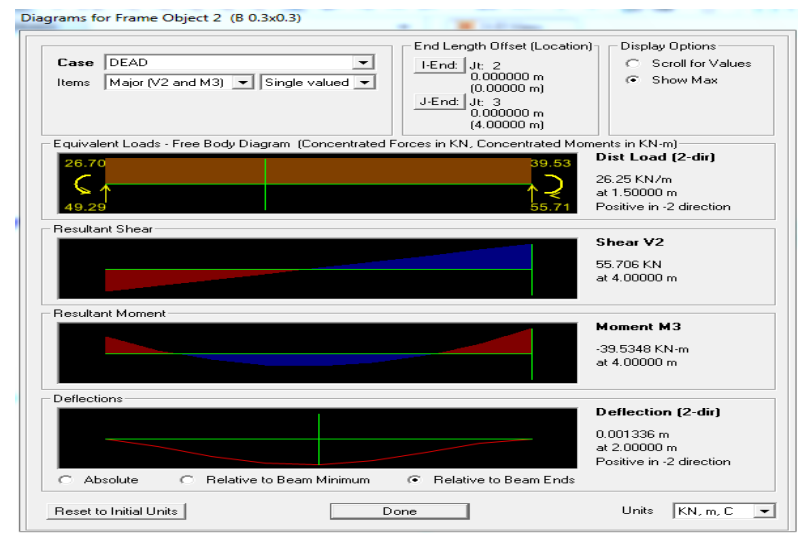

Fig.17 (a) SF, B.M. \& deflection output in SAP-2000

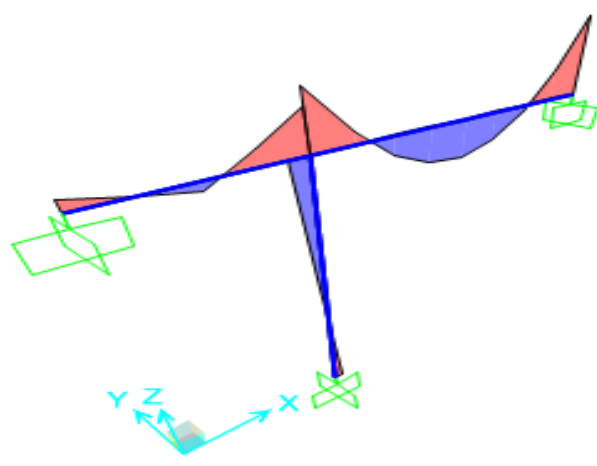

Fig.17 (b) Graphical representation of B.M.\& S.F. in SAP-2000

ETABS $[4,7]$ can do concrete frame design, steel frame design, steel joist design, composite beam design and shear wall design by calculating requirement for overturning and shear in the wall. The design of ETABS is directly displayed on the members of the structure, which is of great help to the designer. ETABS has special feature of bar arrangement as the input to get the realistic and economical design. Design of any arbitrary shape column is possible using this software. Fully interactive design overwrites changes the results instantaneously in the graphical display when property of any of the member is changed without re-analysis and redesign. The shear force, bending moment and deflection are shown in Figure 18(a) and Figure 18(b). ETABS is used in the structure design of building such as Bruj Khalifa, China TV tower.

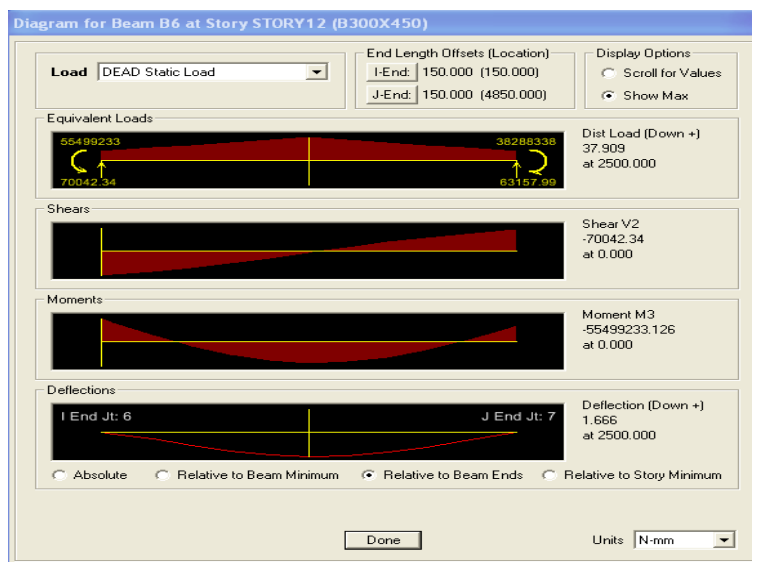

Fig. 18(a) S.F., B.M. \& deflection output in ETABS

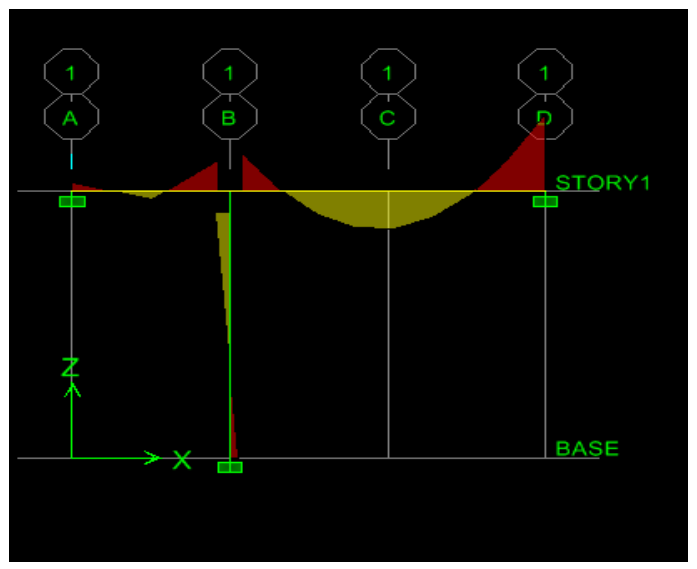

Fig. 18(b) Graphical representation of B.M. \& S.F. in ETABS 


\section{G. Design Output}

STAAD Pro $[2,6]$ gives the output in the form of output file which can be printed by attaching printer to the computer as shown in Table 1(a) and Table 1(b). The STAAD Pro text file (file extension.ANL) contains the printable output data as per the manual. The output is stored in different extension files (file extension.ANL, file extension.BMD, file extension.TMH etc.) .The bending moment shear force, axial force, equivalent force is available by simply clicking on the member at the centre line of elements.

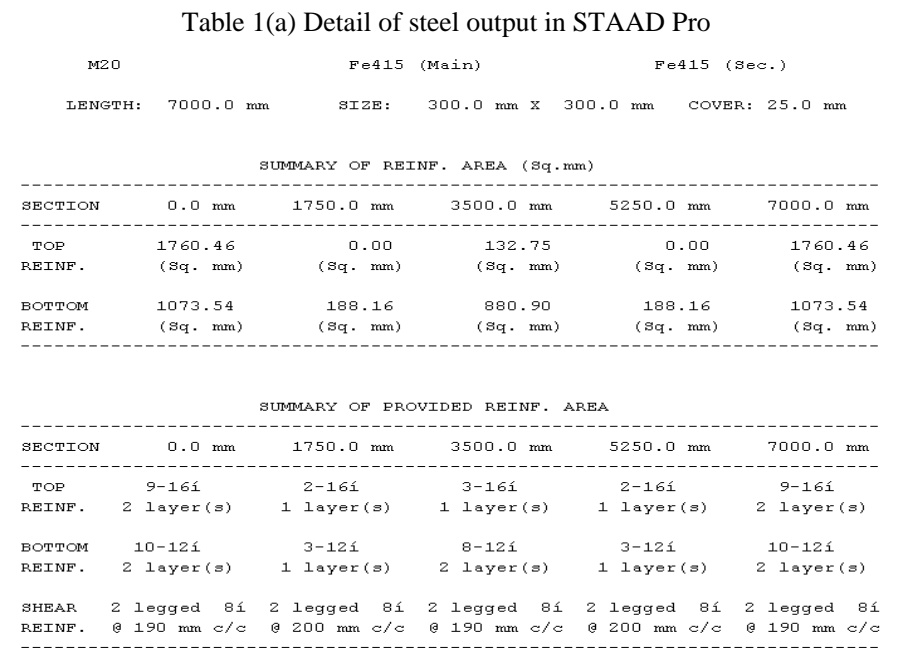

Table 1(b) Output total quantity of steel and concrete used in STAAD Pro.

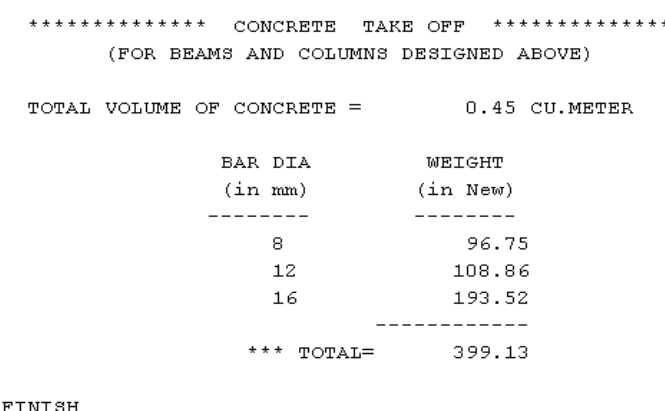

The SAP-2000 [3, 7] output is available in the form of 3D perspectives of deformed and un-deformed shape. Animation of different mode shapes, deformed shapes can be seen in different output parameters by multiple windows. The size of beam, column and area of steel are directly displayed on the frame members in concrete structure as shown in the Figure 19. For steel structure the section size and shape are directly displayed on each member of a structure.

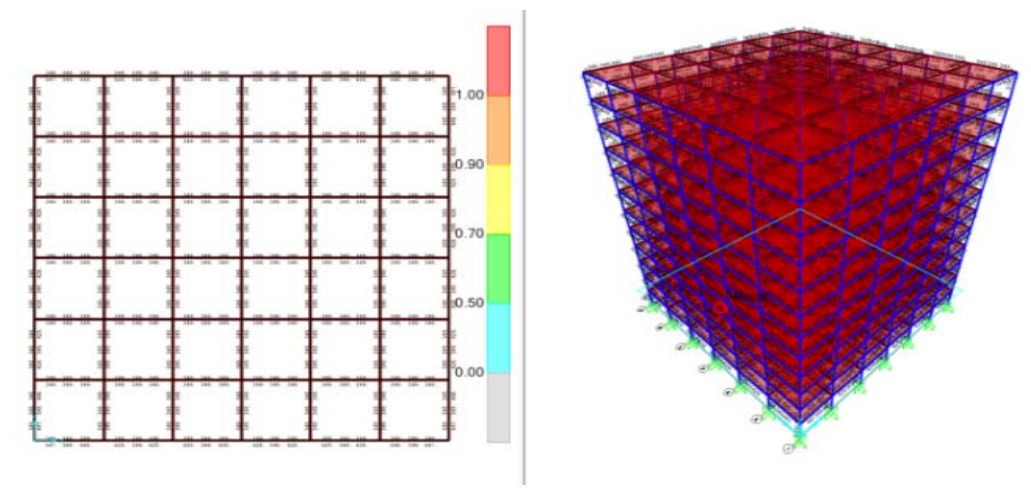

Fig.19 Detail of steel output in SAP-2000 
ETABS $[4,7]$ gives output in the form of 3D deformed geometry shown in Figure 20. After analysis, moment, shear and axial force diagram are given by just clicking on the element member. Different mode shapes and how the structure behaves during horizontal forces can be viewed through animation video. The video of time history response is stored as etabs.avi file. The analysis data in ETABS is stored in etabs.log file. The new model can be initialized using any etabs.edb file. The values of steel reinforcement for the beam are based on the moment at column face and reinforcement for the column is based on the moment at the beam soffit. For both cases the reinforcement in ETABS software program is calculated based on faces rather than at the centerline which is more practical and realistic than the values at the centre line given by STAAD Pro and SAP-2000 software program.

The output design results in ETABS and SAP-2000 are automatically revised without re-analysis but in STAAD Pro re-analysis is required every time the input parameter is changed. One of the drawback of ETABS and SAP2000 is that user cannot read or take printout of the input file easily as compared to STAAD Pro.

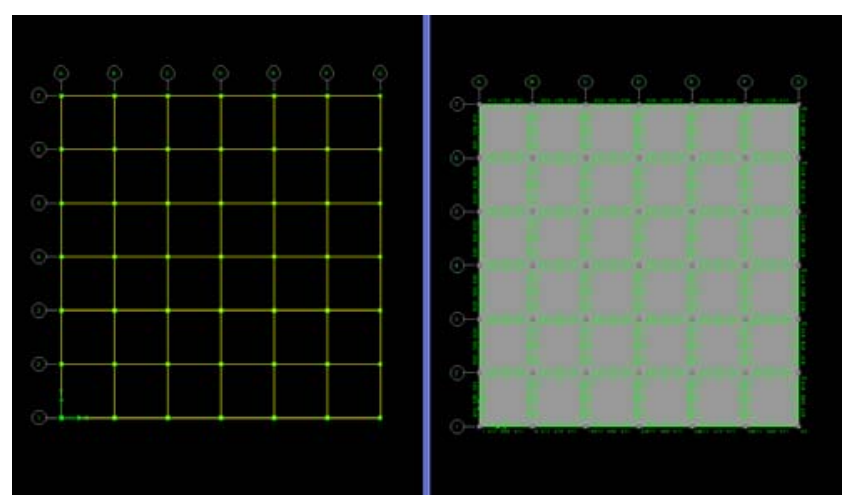

Figure 20 Detail of steel output in ETABS

The output design results in ETABS and SAP-2000 are automatically revised without re-analysis but in STAAD Pro re-analysis is required every time the input parameter is changed. One of the drawback of ETABS and SAP-2000 is that user cannot read or take printout of the input file easily as compared to STAAD Pro.

\section{H. System Requirements}

STAAD Pro [2, 6] requires computer having operating system Microsoft Widow Vista, XP or Window-2000 with minimum RAM of 128MB, 200MB free hard space with graphic card supported by DirectX9.0.The processor of the computer should be higher than Intel Pentium or AMD Athlon.

SAP-2000 [3] requires computer having operating system Microsoft Window Vista, Microsoft window 7 or Microsoft Window 8 having 32bit or 64 bit version. The disk required for the installation of the software is 6GBwith recommended 500GB or larger hard disk drive with speed of $7200 \mathrm{rpm}$ SATA. Additional space is required for the running and storing the model files and the analysis results dependent on size of model. Video card having minimum resolution of 1025 by 768bwith 16 bit colors having Ram of 512MB is required. Minimum Intel Pentium 4 or AMD Athlon64 is required.

ETABS [4] requires computer having operating system Microsoft Window XP with service pack-2 or later, Microsoft Window 8 having 32bit or 64 bit version. The disk required for the installation of the software is 6GB with recommended 500GB or larger hard disk drive with speed of $7200 \mathrm{rpm}$ SATA. Additional space is required for the running and storing the model files and the analysis results dependent on size of model. Video card having minimum resolution of 1025 by 768bwith 16 bit colors having Ram of 512MB is required. Minimum Intel Pentium 4 or AMD Athlon64 is required.

\section{FUTURE SCOPE}

From the above discussions, it is evident that STAAD pro incorporates well provisions from various standards and therefore the results accordingly are more accurate. However, SAP and ETAB provides more flexibility in modeling the structures and in the design details. There are reports that these software many a times provides results which do not confirm the requirements of codal provisions. Such limitations of every software need to be explored and rectified for better confidence of the designer. Further, the software can be made more users friendly. 


\section{REFERENCES}

[1] Kohnke P, editor. ANSYS, Inc. Theory: Release 5.7. Ansys; 2001.

[2] Bentley System Inc. Bentley System Official Website 2014, https://www.bentley.com.

[3] Computer and Structures Inc. (2007), SAP2000, Berkeley, CA, U.S.A.

[4] Computer and Structures Inc. (2003), ETABS, Berkeley, CA, U.S.A.

[5] Fu F. Advanced Modeling Techniques in Structural Design, John Wiley \& Sons: Jun 2015.

[6] Technical Reference Manual, Bentley Inc. (2012), STAAD Pro V8i, Stockton, Exton, U.S.A.

[7] Wilson EL. Three-dimensional Static and Dynamic Analysis of Structures. Computers and Structures, Inc., Berkeley, CA. 1996.

[8] Structural Engineering Forum of India, http://www.sefindia.com. Date accessed: 03/03/2017.

[9] Abaqus 6.9 User Documentation, Internet Manual. Simulia. Retrieved 10 September 2011.

\section{AUTHORS PROFILE}

Er. Balwinder Lallotra is a Research Scholar at PTU, Jalandhar, India and working at M. M. University at Ambala as an Associate Professor. He has about 20 years experience both in consultancy relates to structural design and teaching. The other interests of author are computer aided design, remote sensing and GIS.

Dr Dhirendra Singhal is a Professor and Chairperson in Department of Civil Engineering at D.C.R. University of Science and Technology, Murthal, India. He has number of papers to his credit in reputed journals and has been awarded for the best research paper by the Institution of Engineers (India). He is a member of various technical organisations and associated with various research projects. 